OPEN ACCESS

Edited by: Simone Brogi,

University of Pisa, Italy

Reviewed by:

Jun-Seok Lee,

Korea University, South Korea

Clemens Zwergel,

Sapienza University of Rome, Italy Reshma Rani,

Amity University, India Chiara Borsari,

University of Basel, Switzerland

${ }^{*}$ Correspondence:

Adalberto Fernandes Santos adalberto.santos@ubi.pt

Specialty section: This article was submitted to Medicinal and Pharmaceutical Chemistry,

a section of the journal

Frontiers in Chemistry

Received: 24 March 2021

Accepted: 31 August 2021 Published: 10 September 2021

Citation:

Santos AF, Póvoa $P$, Paixão $P$, Mendonça $A$ and Taborda-Barata $L$ (2021) Changes in Glycolytic Pathway in SARS-COV 2 Infection and Their Importance in Understanding the

Severity of COVID-19.

Front. Chem. 9:685196.

doi: $10.3389 /$ fchem.2021.685196

\section{Changes in Glycolytic Pathway in SARS-COV 2 Infection and Their Importance in Understanding the Severity of COVID-19}

\author{
Adalberto Fernandes Santos ${ }^{1,2,3 *}$, Pedro Póvoa ${ }^{4,5,6}$, Paulo Paixão ${ }^{5,7}$, António Mendonça ${ }^{2,8}$ \\ and Luís Taborda-Barata ${ }^{1,2,9}$
}

${ }^{1}$ Faculty of Health Sciences, University of Beira Interior, Covilhã, Portugal, ${ }^{2} \mathrm{CICS}-U B I$ Health Sciences Research Centre, Universidade da Beira Interior, Lisbon, Portugal, ${ }^{3}$ Department of Teaching and Research of Biochemistry, Faculty of Medicine, Agostinho Neto University, Luanda, Angola, ${ }^{4}$ Polyvalent Intensive Care Unit, Centro Hospitalar de Lisboa Ocidental, Hospital de Sao Francisco Xavier, Lisbon, Portugal, ${ }^{5}$ Comprehensive Health Research Center-CHRC, NOVA Medical School, Universidade Nova de Lisboa, Lisbon, Portugal, ${ }^{6}$ Center for Clinical Epidemiology and Research Unit of Clinical Epidemiology, OUH Odense University Hospital, Odense, Denmark, 'Laboratório de Patologia Clínica-SYNLAB, Hospital da Luz, Lisbon, Portugal,

${ }^{8}$ Department of Chemistry, Faculty of Sciences, University of Beira Interior, Covilhã, Portugal, ${ }^{9}$ Department of Immunoallergology, Cova da Beira University Hospital Centre, Covilhã, Portugal

COVID-19 is an infectious disease caused by Coronavirus 2 (SARS-CoV-2) that may lead to a severe acute respiratory syndrome. Such syndrome is thought to be related, at least in part, to a dysregulation of the immune system which involves three main components: hyperactivity of the innate immune system; decreased production of type 1 Interferons (IFN) by SARS-CoV-2-infected cells, namely respiratory epithelial cells and macrophages; and decreased numbers of both $\mathrm{CD} 4^{+}$and particularly $\mathrm{CD} 8^{+} \mathrm{T}$ cells. Herein, we describe how excessive activation of the innate immune system and the need for viral replication in several cells of the infected organism promote significant alterations in cells' energy metabolism (glucose metabolism), which may underlie the poor prognosis of the disease in severe situations. When activated, cells of the innate immune system reprogram their metabolism, and increase glucose uptake to ensure secretion of proinflammatory cytokines. Changes in glucose metabolism are also observed in pulmonary epithelial cells, contributing to dysregulation of cytokine synthesis and inflammation of the pulmonary epithelium. Controlling hyperglycolysis in critically ill patients may help to reduce the exaggerated production of pro-inflammatory cytokines and optimise the actions of the adaptive immune system. In this review, we suggest that the administration of non-toxic concentrations of 2-deoxy-D-glucose, the use of GLUT 1 inhibitors, of antioxidants such as vitamin $\mathrm{C}$ in high doses, as well as the administration of $\mathrm{N}$-acetylcysteine in high doses, may be useful complementary therapeutic strategies for these patients, as suggested by some clinical trials and/ or reports. Overall, understanding changes in the glycolytic pathway associated with COVID-19 infection can help to find new forms of treatment for this disease.

Keywords: glycolytic pathway, COVID-19, oxidative phosphorylation, reactive oxygen species, hyperglycolysis 


\section{INTRODUCTION}

COVID-19 is an infectious disease that emerged in December 2019 in Wuhan City, China (Zhu et al., 2020). It is caused by Coronavirus 2 Severe Acute Respiratory Syndrome (SARS-CoV-2) and, although most infected individuals are asymptomatic, severe acute respiratory syndrome may develop, and be related, at least in part, to a dysregulation of the immune system (Mehta et al., 2020; Ye et al., 2020). While still controversial in its details, such dysregulation is believed to involve at least three major components.

The first component concerns a hyperactivated state of the innate immune system, which plays a fundamental role in the progression of the disease towards more severe forms and a reserved prognosis. A similar situation was also observed in the 2002-2004 SARS and in the 2012-2015 Middle East Respiratory Syndrome (MERS) epidemics (Gralinski and Baric, 2015; Lu et al., 2020). In this context, there is production of several proinflammatory cytokines which induce persistent inflammation, mainly in the lung (Paces et al., 2020). Most researchers consider this cytokine dysregulation a "cytokine storm" given the higher-than-normal levels of detected cytokines (Mehta et al., 2020; Ye et al., 2020). However, although cytokines such as IL-6 are increased in COVID-19, their serum levels are clearly lower than in Acute Respiratory Distress Syndrome (ARDS) from other causes, and lower than expected in severe cases of COVID-19 (Azoulay et al., 2020; Sinha et al., 2020; Tang et al., 2020).

The second component involves a decrease, or, at least a significant delay, in production of type 1 Interferons (IFN) by SARS-CoV-2-infected cells, namely respiratory epithelial cells and macrophages (Jafarzadeh et al., 2020; Kumar, 2020; Merad and Martin, 2020). Such a deficiency in type 1 IFN is crucial to progression of COVID-19 disease since the anti-viral role of this type of interferons is essential to dealing with the infection (Jafarzadeh et al., 2020; Paces et al., 2020; Gustine and Jones, 2021).

The final component observed in more severe cases of COVID19 involves decreased numbers of both $\mathrm{CD}^{+}$and particularly $\mathrm{CD}^{+} \mathrm{T}$ cells (Leonardi and Proença, 2020), which may also be dysfunctional and, in the latter cells, also involve hypercytotoxic states (Chen et al., 2020a; Qin et al., 2020; Schultheiss et al., 2020). In addition, decreased numbers of Natural Killer (NK) cells in peripheral blood can also be observed, which further weakens antiviral responses, since $\mathrm{CD}^{+} \mathrm{T}$ cells and $\mathrm{NK}$ cells are the main drivers of anti-viral cellular immune responses (Merad and Martin, 2020; Paces et al., 2020).

This mini-review takes into account the three components mentioned above and will aim at putting forward the concept that changes in the glycolytic pathway that occur during SARS-CoV-2 infection significantly contribute to the immune dysregulation observed in severe COVID-19 respiratory infections and also that specific drug treatments that interfere with such changes in the glycolytic pathway may be clinically beneficial in infected patients.

\section{THE GLYCOLYTIC PATHWAY}

The glycolytic pathway is a metabolic pathway of foremost importance in supporting life in almost all organisms (aerobic or anaerobic). It occurs in the cytosol and, under aerobic conditions, aims to convert the active form of glucose (glucose-6-phosphate) $\left(\mathrm{C}_{6} \mathrm{H}_{11} \mathrm{O}_{9} \mathrm{P}\right)$ into pyruvate $\left(\mathrm{C}_{3} \mathrm{H}_{4} \mathrm{O}_{3}\right)$ (Li et al., 2018) (Figure 1). In this pathway, there is cleavage of glucose $\left(\mathrm{C}_{12} \mathrm{O}_{6} \mathrm{H}_{12}\right)$, resulting in the production of potentially energetic intermediates such as pyruvate and NADH $\left(\mathrm{C}_{21} \mathrm{H}_{29} \mathrm{~N}_{7} \mathrm{O}_{14} \mathrm{P}_{2}\right)$, as shown in the following equation " $\underline{\mathrm{C}}_{6} \underline{\mathrm{H}}_{12} \underline{\mathrm{O}}_{6}+2 \mathrm{ADP}+2 \mathrm{Pi}+2 \mathrm{NAD}+\rightarrow 2 \underline{\mathrm{C}}_{3} \underline{\mathrm{H}}_{4} \underline{\mathrm{O}}_{3}+2 \mathrm{ATP}+$ $2 \overline{\mathrm{NADH}}+2 \mathrm{H}^{+”}$ (Chen et al., 2018; Dunn et al., 2016). These intermediates are subsequently used in the mitochondria, in the tricarboxylic acid (TCA) cycle for the cell respiration process and oxidative phosphorylation (OXPHOS), which in turn produces other intermediates that are used in the electron transport chain for production of large amounts of adenosine triphosphate (ATP) $\left(\mathrm{C}_{10} \mathrm{H}_{16} \mathrm{~N}_{5} \mathrm{O}_{13} \mathrm{P}_{3}\right)$, which is the most important form of chemical energy for the cell (and for life) (Figure 1) (Chen et al., 2018). At the end of the glycolytic pathway, only two molecules of ATP are produced per glucose molecule, whereas through OXPHOS the yield rises to 32 molecules of ATP per glucose molecule (Ostroukhova et al., 2012; Dunn et al., 2016). Under aerobic conditions, the pyruvate produced in the glycolytic pathway is transported into the mitochondria and by the action of the pyruvate dehydrogenase enzyme complex, it is converted into acetyl $\mathrm{CoA}\left(\mathrm{C}_{23} \mathrm{H}_{38} \mathrm{~N}_{7} \mathrm{O}_{17} \mathrm{P}_{3} \mathrm{~S}\right)$, which is used in oxidative phosphorylation to produce important intermediates for the electron transport chain (Dunn et al., 2016). Oxygen is used as the final electron acceptor at the end of the electron transport chain, and part of this oxygen is transformed into reactive oxygen species (ROS) by mitochondria, which are the main generators of ROS, even in a physiological context (Ostroukhova et al., 2012).

In hypoxia situations, changes occur in the energy metabolism of cells in general, due to the lack or decrease of oxygen supply. In particular, pyruvate that was converted to acetyl $\mathrm{CoA}$ is now reduced into lactate $\left(\mathrm{C}_{3} \mathrm{H}_{5} \mathrm{O}_{3}\right)$ by the action of the lactate dehydrogenase (LDH) enzyme (Figure 1) (Ostroukhova et al., 2012). This means that the production of ATP by OXPHOS is compromised (Ostroukhova et al., 2012), and the decrease in intracellular oxygen supply reduces the production of ATP by the electron transport chain. Thus, in hypoxia, cells can only rely on the glycolytic pathway, which produces just two molecules of ATP per glucose molecule, a situation that explains the greater consumption of glucose (hyperglycolysis) as the main source of energy production (Dunn et al., 2016). It should be highlighted that hyperglycolysis, that occurs in such cellular hypoxia situations, is due to reprogramming in the metabolism of glycolysis, and is characterised both by the production of ATP exclusively via the glycolytic pathway, and by inhibition of oxidative phosphorylation (Figure 1) (Li and Zhang, 2016). Hyperglycolysis or metabolic reprogramming may also occur in normoxic situations, namely when there is a need for the immediate use of glucose by cells, in order to increase their performance in carrying out tasks such as proliferation, activation, or synthesis of molecules. This strategy is known as aerobic glycolysis, since the increase in glucose consumption happens in the presence of oxygen ( $\mathrm{Li}$ and Zhang, 2016).

In the situations involving metabolic reprogramming described above, there is thus an enzymatic redefinition that 


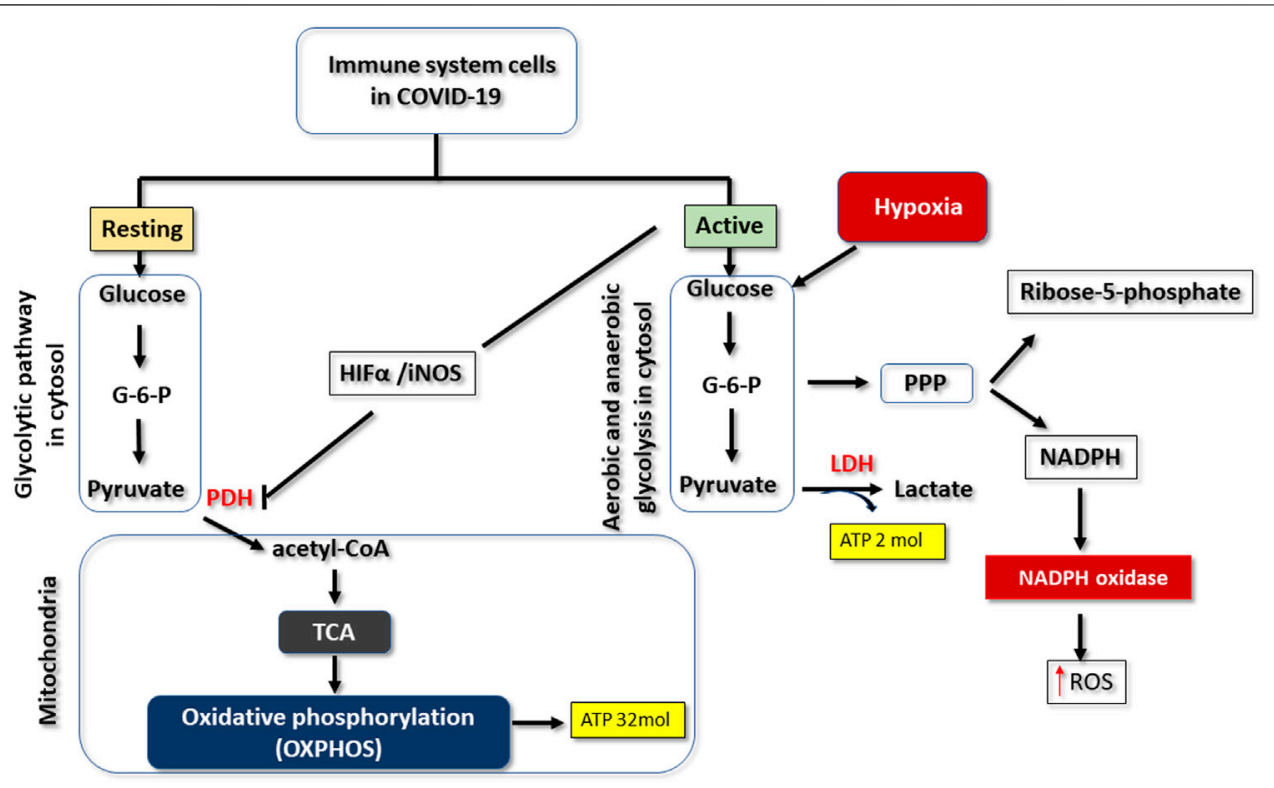

FIGURE 1 | Changes that can occur in the glycolytic pathway in COVID-19. When immune cells are resting, glucose is metabolised via the glycolytic pathway, yielding pyruvate as a product. This substrate is subsequently used in the tricarboxylic acid cycle (TCA) to form intermediates that will be processed by oxidative phosphorylation to produce 32 molecules of ATP, for each molecule of glucose. When immune cells are activated or hypoxic, glucose metabolism is reprogrammed. There is an increase in the type 1 alpha hypoxia-inducing factor ( $\mathrm{HIFa})$, which inhibits pyruvate dehydrogenase enzyme complex (PDH). Pyruvate is thus transformed into lactate by the action of lactate dehydrogenase (LDH). But this mechanism motivates the capture of more glucose for the synthesis of further ATP molecules. There is also activation of the pentose phosphate (PPP) pathway that produces ribose and NADPH. The latter can be used by immune cells through the actions of NADPH oxidase to produce reactive oxygen species (ROS).

involves decreased pyruvate dehydrogenase activity and increased lactate dehydrogenase (LDH) levels (Figure 1) (Seheult et al., 2017). In such metabolic reprogramming, increased expression of hypoxia-1 alpha-inducing factor (HIF1a) protein contributes to the previously mentioned decreased activity of pyruvate dehydrogenase by inhibiting the pyruvate dehydrogenase complex (Andrejeva and Rathmell, 2017; Kumar, 2020). This explains the inhibition of oxidative phosphorylation, giving rise to aerobic glycolysis or hyperglycolysis (Figure 1) (Bergsneider et al., 1997; Andrejeva and Rathmell, 2017; Kumar, 2020).

In inflammatory and septic conditions, as happens in a patient with severe COVID-19, there is also reprogramming of glucose metabolism, associated with increased expression of the ubiquitous sodium-independent glucose transporter 1 (GLUT1) in immune and non-immune cells, increased expression of glycolytic enzymes, such as phosphofructokinase-2, as well as inhibition of pyruvate dehydrogenase enzyme complex by increasing the expression of HIF-1a, which contributes to the increase in glucose uptake (Icard et al., 2021). These hyperglycolytic events that occur in response to stress (i.e., infection-induced stress) also contribute to viral replication and worsening of inflammation (Kumar, 2020). Thus, the inhibition of aerobic hyperglycolysis in SARS-COV-2infected cells may help to control viral replication and decrease inflammation, as has been shown in some in vitro studies in which nontoxic concentrations of 2-deoxy-D-glucose inhibited viral replication in human, colon carcinoma-derived Caco-2 cells (Bojkova et al., 2020).

\section{INCREASED GLUCOSE CONSUMPTION BY CELLS IN SARS-COV-2 INFECTION}

SARS-CoV-2 binds via its spike (S) protein to receptors for the angiotensin-converting enzyme 2 (ACE2) on target cells, and this protein: receptor complex is internalised by cells, namely respiratory epithelial or innate immune system cells (Zhang et al., 2020a). In infected cells, SARS-CoV-2 can induce a set of changes at various levels, namely considerable changes in the glycolytic pathway (Ding et al., 2017; Alfano et al., 2021) (Figure 2). Various mechanisms contribute to this.

First, once inside a cell, the presence of SARS-CoV-2 activates mechanisms, namely mitochondrial oxidative damage, that upregulate intracellular production of ROS, which, in turn, increase cellular injury and cause intracellular stress thereby forcing the infected cell to have increased glucose concentrations. Thus, these cells reprogram their glucose metabolism, and this may involve changes in the final product of the glycolytic pathway (production of lactate instead of pyruvate), activation and inhibition of enzymes (activation of lactate dehydrogenase, and inhibition of pyruvate dehydrogenase) that favour the infectious scenario (Saleh et al., 2020). COVID-19 infection-associated intracellular stress increases endoplasmic reticulum (ER) stress which has been found to be associated with increased expression of GlucoseRegulated Protein 78 (GRP78) at the membrane level, in various settings (e.g., cancer, obesity) (Pfaffenbach and Lee, 2011). Increased levels of GRP78 are not only important for reducing 


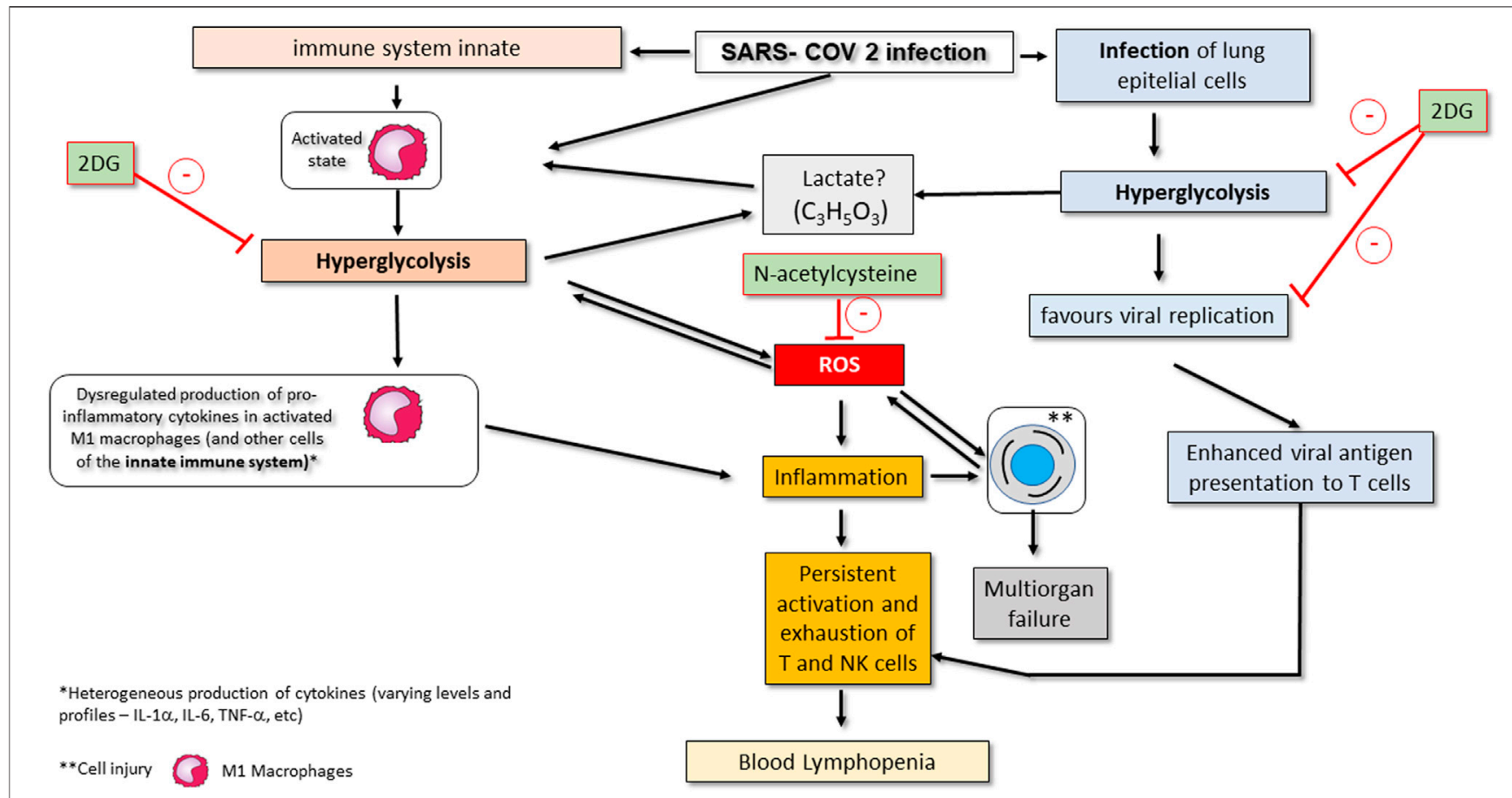

FIGURE 2 | Conceptual model of SARS-CoV-2-associated changes in the glycolytic pathway and its consequences on COVID-19 in critically ill patients. Viral infection activates the innate immune system, namely M1 macrophages. Activation of macrophages promotes metabolic changes for their effective action, including an increase in consumption of glycolysis (hyperglycolysis) and a change in the strategy for ATP synthesis (metabolic reprogramming). These changes favour the production of ROS and worsen inflammation. Hyperglycolysis may also promote an increase in serum lactate. SARS-CoV-2 infection also affects epithelial lung cells by making them increase their glucose consumption, which makes viral replication viable. Infected epithelial cells also show enhanced presentation of viral antigens to $T$ lymphocytes (particularly CD8 ${ }^{+} \mathrm{T}$ cells), and NK cells are also affected, with both cell types becoming more chronically activated. These events promote exhaustion and subsequent apoptosis of T and NK cells, thereby contributing to peripheral lymphopenia. Drugs that may eventually be used as complementary treatment of severe COVID-19 infection by inhibition infection-associated hyperglycolysis include 2-deoxy-D-glucose (2DG), N-acetylcysteine or GLUT1 inhibitors. Administration of 2DG inhibits hyperglycolysis in innate immune system cells such as macrophages; on the other hand, the administration of $\mathrm{N}$-acetylcysteine inhibits the increase in ROS production by activating glutathione (see therapeutic strategies section). The action of GLUT1 inhibitors is not shown but involves blocking glucose uptake in activated and infected immune system and epithelial cells.

ER oxidative stress but translocation of this protein to the membrane also aims to meet the increased demand for glucose inside the infected and stressed cell, since GRP78 contributes to increased glucose uptake into cells (Madhavan and Nagarajan, 2020). In fact, elevated GRP78 levels are significantly higher in COVID-19 infected patients with or without pneumonia than in healthy controls and also tend to be higher than in COVID-negative patients with pneumonia (Sabirli et al., 2021).

Second, patients with severe SARS-CoV-2 infection have been shown to have higher levels of lactate dehydrogenase (LDH) in the peripheral blood compared to patients with mild or moderate disease (Chen et al., 2020b; Mo et al., 2020; Tian et al., 2020). For this reason, $\mathrm{LDH}$ is used as one of several relevant parameters to assess the severity of COVID-19 (Zhang et al., 2020b). The augmented levels of LDH in COVID-19 are related to an increase in the production of lactate, which means that changes in the glycolytic pathway and/or low oxygen conditions occur in cells in the context of infection and/or activation (Ding et al., 2017; Strachecka et al., 2019; Farhana and Lappin, 2020; Morassi et al., 2020). In SARS-CoV 2 infection, with abrupt lung involvement, there is a decrease in oxygen supply in lung cells due to inflammatory events resulting from the infection (Jahani et al., 2020; Serebrovska et al., 2020; Ye et al., 2020) This situation leads to a set of changes in the glycolytic pathway, as well as increased glucose uptake, that impair cell function, thereby worsening events that eventually lead to multiorgan dysfunction, as increased glucose uptake promotes ideal conditions for an inflammatory environment and ROS production (Paces et al., 2020).

\section{CONSEQUENCES OF GLUCOSE METABOLISM REPROGRAMMING IN INFECTED LUNG EPITHELIAL CELLS}

Pulmonary epithelial cells infected by SARS-COV 2 also have an increased expression of HIF-1 a which is closely associated with metabolic reprogramming (Kumar, 2020). In these cells, metabolic reprogramming increases consumption of glucose (Figure 2) and as a consequence, it augments local secretion of proinflammatory cytokines (Neufeldt et al., 2020) that are responsible for the infiltration of monocytes and neutrophils into the lungs. On the other hand, the virus inhibits the secretion of 
type 1 IFN in human airway epithelial cells (Vanderheiden et al., 2020), which reduces antiviral mechanisms and may also contribute to worsening of the inflammatory and septic condition (Kumar, 2020).

In general, in severe situations of SARS-CoV infection, metabolic reprogramming in human airway epithelial cells promotes increased glucose uptake and increased oxidative stress that culminates in increased lactate production, and mitochondrial damage, as well as dysregulation of the innate immune system (including overactivation and functional dysregulation of the innate immune system, as well as functional depletion and dysregulation of $\mathrm{T}$ cells), which results in a poor prognosis in critically ill patients (Li et al., 2018).

\section{CHANGES IN THE GLYCOLYTIC PATHWAY IN IMMUNE CELLS IN SARS-COV-2 INFECTION}

Some in vitro studies have shown that cultures of SARS-CoV-2infected bronchoalveolar lavage monocytes from patients with COVID-19 have upregulated expression of genes associated with glycolysis (Codo et al., 2020). In addition, the same study also showed that SARS-CoV-2 infection, but not infection with the human H1N1 influenza A or respiratory syncytial virus, specifically stimulated glycolysis and increased glycolytic capacity and glycolytic reserve in monocytes (Codo et al., 2020).

The production of cytokines in immune cells such as macrophages, dendritic cells or neutrophils, as well as activation of these cells is intrinsically linked to reprogramming of energy metabolism (Figure 2) (Patel et al., 2019; Codo et al., 2020), and this also occurs in SARS-CoV-2 infection (Kumar, 2020). Activated macrophages undergo metabolic reprogramming, by switching from oxidative phosphorylation to aerobic glycolysis, a strategy that proves to be more profitable and effective for ATP production required for the secretion of cytokines and all other effector actions that these cells need to perform in the case of infection, namely antigen presentation to $\mathrm{T}$ cells (Icard et al., 2021).

Thus, SARS-CoV-2 infection activates the innate immune system and, if this process becomes uncontrolled, it can result in the dysregulation of cytokine production (Figure 2), involving increased production of pro-inflammatory cytokines, namely interleukin-6 (IL-6), IL-8, TNF- a and others, which contribute to the development of ARDS (Fu et al., 2020). In the context of ARDS it is accepted that there is a decrease in tissue oxygen which, if severe, is reflected in cellular hypoxia, thereby further contributing to metabolic reprogramming in immune cells (Jahani et al., 2020; Serebrovska et al., 2020).

However, in some situations, the alteration in the glycolytic pathway does not occur towards production of aerobic glucose, which induces a more efficient immune response, but rather towards oxidative phosphorylation, which compromises and delays this response. In this sense, the virus inhibits molecular signaling mechanisms that activate aerobic glycolysis, thereby impairing the synthesis of type 1 IFN and contributing towards dysregulated production of pro-inflammatory cytokines, a scenario observed not only in infected epithelial cells, but also in infected dendritic cells and peripheral blood mononuclear cells (Gardinassi et al., 2020).

Furthermore, persistent inflammation mediated by the innate immune system, namely M1 macrophages, as well as the constant presentation of viral antigens to $\mathrm{CD} 8^{+}$cytotoxic $\mathrm{T}$ cells contribute to the exhaustion of the latter cells (Figure 2), and, as consequence, their energy metabolism also changes from aerobic glucose to oxidative phosphorylation (Fenwick et al., 2019; Kumar, 2020). In fact, in infected cytotoxic CD8 ${ }^{+}$ $\mathrm{T}$ cells, the production of ATP ends up being provided by oxidative phosphorylation, in contrast to aerobic glycolysis. This situation is detrimental to infected cells due to the fact that they produce more ROS via mitochondria, may become more dysfunctional and enter apoptosis (Fenwick et al., 2019).

\section{WILL THERE BE LACTIC ACIDOSIS IN SARS-COV-2 INFECTION DUE TO ALTERATION OF THE GLYCOLYTIC PATHWAY?}

Changes in glucose metabolism that promote hyperglycolysis lead to the formation of significant amounts of lactate (Figure 1) (Brooks, 2018). Lactate production can have differential effects on immune cells, namely indirect inhibition of production of type 1 IFN in macrophages (Kumar, 2020; Zhang et al., 2019). These effects would also be expected in patients with SARS-CoV-2 infection, because of the changes in the glycolytic pathway that promote overactivation of the innate immune system (Figure 2) (Fenwick et al., 2019; Ye et al., 2020). High levels of lactate could also be expected due to pulmonary impairment in critically ill patients with SARS-CoV-2. However, lactate does not seem to be associated with a poor prognosis and progression of the disease, since its serum levels are not increased in the overwhelming majority of critically ill patients with COVID-19 (Mo et al., 2020; Tang et al., 2020). Recently, a retrospective study that aimed to identify acid-base disorders in patients with COVID-19 identified only one patient whose metabolic acidosis was caused by a slight increase in peripheral blood lactate, in a sample of 211 patients (Alfano et al., 2021). In fact, the vast majority of studies do not report such a condition in patients with severe COVID-19 infection who are hospitalized in ICU wards, even when LDH levels are increased (Zhang et al., 2019). This is an interesting situation that could lead to a discussion about the effects of hypoxia and hyperglycolysis in COVID-19 from a metabolic point of view, and raise the question of why there is no detectable lactic acidosis in the peripheral blood of critically ill patients with COVID-19. The mechanism behind the lack of an increase in lactate levels is not known, but one hypothesis may be that produced lactate can be rapidly consumed by the lactateproducing cell as it is used, for instance, for binding to mitochondrial antiviral-signaling protein (MAVS) which may result in decreased type 1 IFN production as was seen in HEK-293 human kidney cell lines (Zhang et al., 2019). On the other hand, there is also the possibility that the non-accumulation of lactate in 
the bloodstream may be due to its consumption by the liver and kidneys, through the Cori cycle, allowing the production of more glucose. The increase in LDH could reinforce this idea, since this enzyme is also involved in the conversion of lactate to pyruvate in the Cori cycle (Marik and Bellomo, 2013; Gurung and Jialal, 2020; Levitt et al., 2020; Yang et al., 2020).

\section{CHANGES IN THE GLYCOLYTIC PATHWAY PROMOTE REACTIVE OXYGEN SPECIES PRODUCTION IN SARS-COV-2 INFECTION}

As was previously mentioned, hyperglycolysis leads to overproduction of ROS (Figure 2). Several cells in different organs, as well as macrophages and neutrophils have an enzyme complex called NADPH oxidase (Figure 1) (Baillet et al., 2017) Among other functions, this transmembrane complex produces ROS, thereby acting as a relevant source of such oxygen radicals in infectious processes (Baillet et al., 2017). $\mathrm{NADPH}$ oxidase uses NADPH produced in the pentose phosphate pathway (PPP) as an electron donor for the production of ROS (Figure 1) (Andrejeva and Rathmell, 2017; Baillet et al., 2017). As hyperglycolysis promotes an increase in the production of glucose-6-phosphate, an intermediate of the glycolytic pathway that is used in the pentose phosphate pathway for the production of ribose-5-phosphate and NADPH, there will necessarily be an increase in ROS production by NADPH oxidase (Figure 1) (Baillet et al., 2017).

The increase in ROS by activating NADPH oxidase further stimulates hyperglycolysis to produce more ATP, and the production of ATP in turn further activates NADPH oxidase. ATP is used to phosphorylate the components of NADPH oxidase, thus leading to its activation. Therefore, the assembly of the enzyme complex is improved to increase production of ROS (Baillet et al., 2017). In addition, there is a process of mutual activation between hyperglycolysis and NADPH oxidase that is mediated by phosphofrutokinase-2 (Batushansky et al., 2019).

It is generally accepted that these situations of hyperglycosis in COVID-19 infection augment the activation of the immune system and worsen inflammatory response, namely via ROS production (Saleh et al., 2020; Icard et al., 2021). Thus, since there is a considerable increase in ROS in severe COVID-19, and little or no production of natural antioxidants by the body, the use of antioxidants could be part of the therapeutic protocols of critically ill patients with COVID-19. In fact, an epidemiological analysis has recently suggested that an anti-oxidant-rich dietary approach may enhance Nuclear factor (erythroid-derived 2)-like 2 (Nrf-2)-associated antioxidant effect, thereby contributing to mitigation of the severity of COVID-19 infection (Bousquet et al., 2020; Bousquet et al., 2021).

\section{THERAPEUTIC STRATEGIES}

Increased glucose consumption (hyperglycolysis), as part of metabolic reprogramming in which there is enzymatic redefinition, appears to be the basis for severity and poor prognosis in critically ill patients infected with SARS-COV-2. Thus, controlling hyperglycolysis in critically ill patients would help to control the dysregulated or exaggerated production of pro-inflammatory cytokines and optimise the action of the adaptive immune system. In this context, the administration of non-toxic concentrations of 2-deoxyD-glucose (2-DG) may be a strategy for minimizing events resulting from increased glucose consumption. 2-DG is a glucose analogue which inhibits the glycolytic pathway, through the inhibition of phosphoglucoisomerase. Thus, there will be no formation of glucose-6-phosphate, which is a crucial intermediate for the continuity of the glycolytic pathway (Saleh et al., 2020; Verma et al., 2020). This mode of action makes 2-DG a possibly useful drug for inhibition of metabolic reprograming which also occurs in cancer, resulting in decreased glucose consumption and inhibition of the glycolytic pathway in cancer cells (Woodward and Cramer, 1952). Studies carried out in vitro and in murine models showed that 2-DG may induce antitumor $\mathrm{CD}^{+} \mathrm{T}$ cellmediated cytotoxicity and increased production of IFN-g (Sasaki et al., 2021). In addition, 2-DG in association with radiotherapy also has other immune effects by increasing cell membrane expression of molecules that are relevant to antigen presentation (MHC II and CD86) by dendritic cells and reducing production of the pro-inflammatory cytokine TNFa (Farooque et al., 2014). All of these actions may be beneficial in the context of severe COVID-19 infection. However, evidence of clinical benefit is warranted in this context. Although 2-DG monotherapy may need to involve high doses and toxicity, small phase I and phase II clinical trials in patients with glioblastoma showed that combined treatment of 2-DG and radiotherapy was well tolerated, moderately improved survival, and improved quality of life (Mohanti et al., 1996; Singh et al., 2005), although there were problems with study design and definition of endpoints in these studies (Mohanti et al., 1996; Singh et al., 2005; Halder and Mehta, 2021). Thus, various aspects regarding safety and efficacy of 2-DG as complementary treatment both in cancer and in infectious diseases such as COVID-19 still have to be addressed by robust, randomized, double-blind, placebocontrolled phase III clinical trials. Currently, at least one phase II clinical trial aiming at studying safety and efficacy of 2-DG in COVID-19 patients is underway (CTRI/2020/06/ 025,664; Cochrane Central register of Controlled Trials).

On the other hand, pharmacological strategies such as the use of GLUT 1 inhibitors (GLUT-1i) aimed at inhibiting glucose transporters could be evaluated in the treatment of critically ill patients, since there is an increase in the expression of GLUTs in immune and non-immune cells in these patients. In fact, the use of glucose inhibitors as a therapeutic strategy in other infectious diseases (e.g., Zika virus or Human $\mathrm{T}$ cell Leukemia Virus infections) and in various types of cancer cells has been documented in vitro by other authors (Manel et al., 2003; Dominguez Rieg and Rieg, 2019; Lin et al., 2019; Reckzeh and Waldmann, 2020; Cao et al., 2021) but data are necessary in the context of the possible use of GLUT-1i as complementary therapy in severe COVID-19 infection. 
Changes in the glycolytic pathway in critically ill, SARS-COV2 -infected patients lead to increased production of ROS and a decrease in compounds that control such radicals, such as glutathione. Based on this scenario, the use of antioxidants such as vitamin $\mathrm{C}$ in high doses as a therapeutic adjuvant in critically ill patients with COVID-19 has been shown to be a promising strategy. A prospective, randomised double-blind clinical trial with a sample of 308 critically ill COVID-19 patients, demonstrated that the use of high doses of vitamin $\mathrm{C}$ improved lung function and reduced mortality from multiple organ failure. It has been shown, despite the need for further studies, that this strategy would help to reduce ARDS events and multiorgan damage caused by excess ROS from alterations in the glycolytic pathway (Liu et al., 2020).

On the other hand, still with the perspective of mitigating ROS-induced events, another interesting therapeutic approach would be the administration of $\mathrm{N}$-acetylcysteine in high doses. This compound is a reduced glutathione $(\mathrm{GSH})$ intermediate (Suhail et al., 2020). Its use aims to increase GSH (a natural antioxidant) levels in the body, since such levels are decreased in critically ill COVID-19 patients (Bartolini et al., 2021; Karkhanei et al., 2021). Beneficial effects of $\mathrm{N}$-acetylcysteine have been demonstrated in clinical trials in various infections, including influenza and influenza-like infections, namely H1N1 influenza pneumonia or even community acquired pneumonia (De Flora et al., 1997; Zhang et al., 2018). In COVID-19, administration of $\mathrm{N}$-acetylcyteine, alone or together with other types of supplementation may be beneficial in patients with different degrees of severity of SARS-CoV-2 infection, as suggested by a few small-sized, non-controlled clinical interventional studies or case reports (De Flora et al., 2020; Avdeev et al., 2021; Wong et al., 2021), although these results may not apply to patients with SARS-CoV-2-induced ARDS (de Alencar et al., 2021; Taher et al., 2021), and still need to be more robustly analysed in larger, randomised, placebo-controlled studies.

\section{REFERENCES}

Alfano, G., Ferrari, A., Fontana, F., Mori, G., Magistroni, R., Guaraldi, G., et al. (2021). Fc 025Acid Base Disorders in Covid-19. Int. Urol. Nephrol. 36, 1-6. doi:10.1007/s11255-021-02855-110.1093/ndt/gfab145.001

Andrejeva, G., and Rathmell, J. C. (2017). Similarities and Distinctions of Cancer and Immune Metabolism in Inflammation and Tumors. Cel Metab. 26 (1), 49-70. doi:10.1016/j.cmet.2017.06.004

Avdeev, S. N., Gaynitdinova, V. V., Merzhoeva, Z. M., and Berikkhanov, Z. G.-M. (2021). N-acetylcysteine for the treatment of COVID-19 among hospitalized patients. J. Infect. S0163-4453 (21), 00329-337. doi:10.1016/j.jinf.2021.07.003

Azoulay, E., Zafrani, L., Mirouse, A., Lengliné, E., Darmon, M., and Chevret, S. (2020). Clinical phenotypes of critically ill COVID-19 patients. Intensive Care Med. 46 (8), 1651-1652. doi:10.1007/s00134-020-06120-4

Baillet, A., Hograindleur, M. A., El Benna, J., Grichine, A., Berthier, S., Morel, F., et al. (2017). Unexpected function of the phagocyte NADPH oxidase in supporting hyperglycolysis in stimulated Neutrophils: Key Role of 6phosphofructo-2-kinase. FASEB j. 31 (2), 663-673. doi:10.1096/fj.201600720R

Bartolini, D., Stabile, A. M., Bastianelli, S., Giustarini, D., Pierucci, S., Busti, C., et al. (2021). SARS-CoV2 infection impairs the metabolism and Redox function of cellular glutathione. Redox Biol. 45, 102041. doi:10.1016/j.redox.2021.102041

Batushansky, A., Matsuzaki, S., Newhardt, M. F., West, M. S., Griffin, T. M., and Humphries, K. M. (2019). GC-MS metabolic profiling Reveals

\section{CONCEPTUAL MODEL}

A general conceptual model of all COVID-19-associated changes in glycolytic pathways as well as potential points for therapeutic interventions is shown in Figure 2.

\section{CONCLUSION}

Events related to changes in the glycolytic pathway in COVID-19 infection may explain the underlying pathology and severity of some cases of the disease. However, although there is clear scientific evidence showing changes in glycolysis in COVID-19 infection, there is still little evidence linking such changes to worse prognosis of COVID-19. Thus, the current review clearly shows that such an association should be considered in future research in this context.

Furthermore, there seems to be a relationship between hyperglycolysis and dysregulation of the immune system, as well as cellular damage. However, further studies are needed to consolidate these ideas and, perhaps, to find therapeutic targets that can mitigate the deleterious events that arise from this context.

Since there is a considerable increase in ROS in severe COVID-19, and little or no production of natural antioxidants by the body, we think that perhaps the use of antioxidants could be part of the therapeutic protocols of critically ill patients with COVID-19.

\section{AUTHOR CONTRIBUTIONS}

AS structured and wrote the mini-review and carried out all relevant searches. AM, PPov, PP, and LT-B helped with writing the mini-review and offered relevant suggestions for improvement.

fructose-2,6-bisphosphate Regulates branched chain amino acid metabolism in the heart during fasting. Metabolomics 15 (2), 18 . doi:10.1007/s11306-019-1478-5

Bergsneider, M., Hovda, D. A., Shalmon, E., Kelly, D. F., Vespa, P. M., Martin, N. A., et al. (1997). Cerebral hyperglycolysis following severe traumatic brain injury in humans: a positron emission tomography study. J. Neurosurg. 86 (2), 241-251. Retrieved Feb 15, 2021, from. doi:10.3171/jns.1997.86.2.024https:// thejns.org/view/journals/j-neurosurg/86/2/article-p241.xml

Bojkova, D., Klann, K., Koch, B., Widera, M., Krause, D., Ciesek, S., et al. (2020). Proteomics of SARS-CoV-2-infected host cells Reveals therapy targets. Nature 583 (7816), 469-472. doi:10.1038/s41586-020-2332-7

Bousquet, J., Anto, J. M., Czarlewski, W., Haahtela, T., Fonseca, S. C., Iaccarino, G., et al. ARIA Group (2020). Cabbage and fermented vegetables: from death Rate heterogeneity in countries to candidates for mitigation strategies of severe COVID-19. Allergy 76, 735-750. doi:10.1111/all.14549

Bousquet, J., Le Moing, V., Blain, H., Czarlewski, W., Zuberbier, T., de la Torre, R., et al. (2021). Efficacy of broccoli and glucoraphanin in COVID-19: From hypothesis to proof-of-concept with three experimental clinical cases. World Allergy Organ. J. 14, 100498. doi:10.1016/j.wa10.1016/j.waojou.2020.100498

Brooks, G. A. (2018). The Science and Translation of Lactate Shuttle Theory. Cel Metab. 27 (4), 757-785. doi:10.1016/j.cmet.2018.03.008

Cao, S., Chen, Y., Ren, Y., Feng, Y., and Long, S. (2021). GLUT1 biological function and inhibition: Research advances. Future Med. Chem. 13, 1227-1243. doi:10.4155/fmc-2021-0071 
Chen, G., Wu, D., Guo, W., Cao, Y., Huang, D., Wang, H., et al. (2020). Clinical and immunological features of severe and moderate coronavirus disease 2019. J. Clin. Invest. 130, 2620-2629. doi:10.11172/JCI13724410.1172/jci137244

Chen, W., Gong, P., Guo, J., Li, H., Li, R., Xing, W., et al. (2018). Glycolysis Regulates Pollen Tube Polarity via Rho GTPase Signaling. Plos Genet. 14 (4), e1007373. doi:10.1371/journal.pgen.1007373

Chen, X.-Y., Huang, M.-Y., Xiao, Z.-W., Yang, S., and Chen, X.-Q. (2020). Lactate dehydrogenase elevations is associated with severity of COVID-19: a metaanalysis. Crit. Care 24, 459. doi:10.1186/s13054-020-03161-5

Codo, A. C., Davanzo, G. G., Monteiro, L. d. B., de Souza, G. F., Muraro, S. P., Virgílio-da-Silva, J. V., et al. (2020). Elevated Glucose Levels Favor SARS-CoV2 Infection and Monocyte Response through a HIF-1 $\alpha /$ Glycolysis-dependent Axis. Cel Metab. 32, 437-446. doi:10.1016/j.cmet.2020.07.007

de Alencar, J. C. G., Moreira, C. L., Müller, A. D., Chaves, C. E., Fukuhara, M. A., da Silva, E. A., et al. (2021). Double-blind, Randomized, Placebo-controlled Trial with $\mathrm{N}$-acetylcysteine for Treatment of Severe Acute Respiratory Syndrome Caused by Coronavirus Disease 2019 (COVID-19). Clin. Infect. Dis. 72, e736-e741. doi:10.1093/cid/ciaal443

De Flora, S., Balansky, R., and La Maestra, S. (2020). Rationale for the Use of N -acetylcysteine in both prevention and adjuvant therapy of COVID-19. FASEB j. 34 (10), 13185-13193. doi:10.1096/fj.202001807

De Flora, S., Grassi, C., and Carati, L. (1997). Attenuation of influenza-like symptomatology and improvement of cell-mediated immunity with longterm N-acetylcysteine treatment. Eur. Respir. J. 10, 1535-1541. doi:10.1183/ 09031936.97.10071535

Ding, J., Karp, J. E., and Emadi, A. (2017). Elevated Lactate Dehydrogenase (LDH) Can Be a Marker of Immune Suppression in Cancer: Interplay between Hematologic and Solid Neoplastic Clones and Their Microenvironments. Cbm 19 (4), 353-363. doi:10.3233/CBM-160336

Dominguez Rieg, J. A., and Rieg, T. (2019). What does sodium-glucose cotransporter 1 inhibition add: Prospects for dual inhibition. Diabetes Obes. Metab. 21 (Suppl. 2Suppl 2), 43-52. doi:10.1111/dom.13630

Dunn, J.-O., Mythen, M., and Grocott, M. (2016). Physiology of Oxygen Transport. BJA Edu. 16 (10), 341-348. doi:10.1093/bjaed/mkw012

Farhana, A., and Lappin, S. L. (2020). Biochemistry, Lactate Dehydrogenase (LDH). StatPearls, 32491468.

Farooque, A., Singh, N., Adhikari, J. S., Afrin, F., and Dwarakanath, B. S. R. (2014). Enhanced antitumor immunity contributes to the Radio-sensitization of Ehrlich ascites tumor by the glycolytic inhibitor 2-deoxy-D-glucose in mice. PLoS One 9, e108131. doi:10.1371/journal.pone.0108131

Fenwick, C., Joo, V., Jacquier, P., Noto, A., Banga, R., Perreau, M., et al. (2019). Tcell exhaustion in HIV infection. Immunol. Rev. 292 (1), 149-163. doi:10.1111/ imr. 12823

Fu, L., Wang, B., Yuan, T., Chen, X., Ao, Y., Fitzpatrick, T., et al. (2020). Clinical characteristics of coronavirus disease 2019 (COVID-19) in China: A systematic Review and meta-analysis. J. Infect. 80 (6), 656-665. doi:10.1016/ j.jinf.2020.03.041

Gardinassi, L. G., Souza, C. O. S., Sales-Campos, H., and Fonseca, S. G. (2020). Immune and metabolic signatures of COVID-19 Revealed by transcriptomics data Reuse. Front. Immunol. 11, 1636. doi:10.3389/fimmu.2020.01636

Gralinski, L. E., and Baric, R. S. (2015). Molecular pathology of emerging coronavirus infections. J. Pathol. 235 (2), 185-195. doi:10.1002/path.4454

Gurung, P., and Jialal, I. (2020). "Plasma Glucose," in StatPearls. Treasure Island (FL) (United States: StatPearls Publishing), 2.

Gustine, J. N., and Jones, D. (2021). Immunopathology of Hyperinflammation in COVID-19. Am. J. Pathol. 191 (1), 4-17. doi:10.1016/j.ajpath.2020.08.009

Halder, S., and Mehta, A. K. (2021). 2-Deoxy-D-glucose: is this the final cure for COVID-19: or yet another mirage?. Eur. Rev. Med. Pharmacol. Sci. 25, 4448-4450. doi:10.26355/eurrev_202107_26234

Icard, P., Lincet, H., Wu, Z., Coquerel, A., Forgez, P., Alifano, M., et al. (2021). The Key Role of Warburg effect in SARS-CoV-2 Replication and associated inflammatory Response. Biochimie 180, 169-177. doi:10.1016/ j.biochi.2020.11.010

Jafarzadeh, A., Chauhan, P., Saha, B., Jafarzadeh, S., and Nemati, M. (2020). Contribution of monocytes and macrophages to the local tissue inflammation and cytokine storm in COVID-19: Lessons from SARS and MERS, and potential therapeutic interventions. Life Sci. 257, 118102. doi:10.1016/ j.lfs.2020.1181010.1016/j.lfs.2020.118102
Jahani, M., Dokaneheifard, S., and Mansouri, K. (2020). Hypoxia: A Key feature of COVID-19 launching activation of HIF-1 and cytokine storm. J. Inflamm. 17 (1), 33. doi:10.1186/s12950-020-00263-3

Karkhanei, B., Talebi Ghane, E., and Mehri, F. (2021). Evaluation of oxidative stress level: total antioxidant capacity, total oxidant status and glutathione activity in patients with COVID-19. New Microbes and New Infections 42, 100897. doi:10.1016/j.nmni.2021.100897

Kumar, V. (2020). How could we forget immunometabolism in SARS-CoV2 infection or COVID-19?. Int. Rev. Immunol. 40, 72-107. doi:10.1080/ 08830185.2020.1840567

Leonardi, A. J., and Proença, R. B. (2020). Akt-Fas to quell aberrant T cell differentiation and apoptosis in Covid-19. Front. Immunol. 11, 600405. doi:10.3389/fimmu.2020.600405

Levitt, D. G., Levitt, J. E., and Levitt, M. D. (2020). Quantitative Assessment of Blood Lactate in Shock: Measure of Hypoxia or Beneficial Energy Source. Biomed. Res. Int. 2020, 1-24. doi:10.1155/2020/2608318

Li, Y., Lu, B., Sheng, L., Zhu, Z., Sun, H., Zhou, Y., et al. (2018). Hexokinase 2Dependent Hyperglycolysis Driving Microglial Activation Contributes to Ischemic Brain Injury. J. Neurochem. 144 (2), 186-200. doi:10.1111/ jnc. 14267

Li, Z., and Zhang, H. (2016). Reprogramming of glucose, fatty acid and amino acid metabolism for cancer progression. Cell. Mol. Life Sci. 73 (2), 377-392. doi:10.1007/s00018-015-2070-4

Lin, S.-C., Chen, M.-C., Liu, S., Callahan, V. M., Bracci, N. R., Lehman, C. W., et al. (2019). Phloretin inhibits Zika virus infection by interfering with cellular glucose Utilisation. Int. J. Antimicrob. Agents 54, 80-84. doi:10.1016/ j.ijantimicag.2019.03.017

Liu, F., Zhu, Y., Zhang, J., Li, Y., and Peng, Z. (2020). Intravenous high-dose vitamin $C$ for the treatment of severe COVID-19: study protocol for a multicentre Randomised controlled trial. BMJ Open 10 (7), e039519. doi:10.1136/bmjopen-2020-039519

Lu, L., Zhong, W., Bian, Z., Li, Z., Zhang, K., Liang, B., et al. (2020). A comparison of mortality-Related Risk factors of COVID-19, SARS, and MERS: A systematic Review and meta-analysis. J. Infect. 81 (4), e18-e25. doi:10.1016/j.10.1016/ j.jinf.2020.07.002

Madhavan, S., and Nagarajan, S. (2020). GRP78 and Next generation cancer hallmarks: An Underexplored molecular target in cancer chemoprevention Research. Biochimie 175, 69-76. doi:10.1016/j.biochi.2020.05.005

Manel, N., Kim, F. J., Kinet, S., Taylor, N., Sitbon, M., and Battini, J.-L. (2003). The Ubiquitous glucose transporter GLUT-1 is a Receptor for HTLV. Cell 115, 449-459. doi:10.1016/s0092-8674(03)00881-x

Marik, P., and Bellomo, R. (2013). Lactate clearance as a target of therapy in sepsis: a flawed paradigm. OA Crit. Care 1 (1), 3. doi:10.13172/2052-9309-1-1-431

Mehta, P., McAuley, D. F., Brown, M., Sanchez, E., Tattersall, R. S., and Manson, J. J. (2020). COVID-19: consider cytokine storm syndromes and immunosuppression. The Lancet 395, 1033-1034. doi:10.1016/S01406736(20)30628-0

Merad, M., and Martin, J. C. (2020). Pathological inflammation in patients with COVID-19: a Key Role for monocytes and macrophages. Nat. Rev. Immunol. 20 (6), 355-362. doi:10.1038/s41577-020-0331-4

Mo, P., Xing, Y., Xiao, Y., Deng, L., Zhao, Q., Wang, H., et al. (2020). Clinical Characteristics of Refractory Coronavirus Disease 2019 in Wuhan, China. Clin. Infect. Dis., 16, 2020. ciaa270. doi:10.1093/cid/ciaa270

Mohanti, B. K., Rath, G. K., Anantha, N., Kannan, V., Das, B. S., Chandramouli, B. A. R., et al. (1996). Improving cancer Radiotherapy with 2-deoxy-D-glucose: Phase I/II clinical trials on human cerebral gliomas. Int. J. Radiat. Oncolog ${ }^{*}$ Biology ${ }^{*}$ Physics 35, 103-111. doi:10.1016/s0360-3016(96)85017-6

Morassi, M., Bagatto, D., Cobelli, M., D’Agostini, S., Gigli, G. L., Bnà, C., et al. (2020). Stroke in Patients with SARS-CoV-2 Infection: Case Series. J. Neurol. 267 (8), 2185-2192. doi:10.1007/s00415-020-09885-2

Neufeldt, C. J., Cerikan, B., Cortese, M., Frankish, J., Lee, J.-Y., Plociennikowska, A., et al. (2020). SARS-CoV-2 infection induces a pro-inflammatory cytokine Response through cGAS-STING and NF-kB. bioRxiv. doi:10.1101/ 2020.07.21.212639

Ostroukhova, M., Goplen, N., Karim, M. Z., Michalec, L., Guo, L., Liang, Q., et al. (2012). The Role of low-level lactate production in airway inflammation in asthma. Am. J. Physiol. Lung Cel Mol Physiol 302 (3), L300-L307. doi:10.1152/ ajplung.00221.2011Ostroukhova 
Paces, J., Strizova, Z., Smrz, D., and Cerny, J. (2020). COVID-19 and the immune system. Physiol. Res. 69 (3), 379-388. doi:10.33549/physiolres10.33549/ physiolres.934492

Patel, C. H., Leone, R. D., Horton, M. R., and Powell, J. D. (2019). Targeting metabolism to Regulate immune Responses in autoimmunity and cancer. Nat. Rev. Drug Discov. 18 (9), 669-688. doi:10.1038/s41573-019-0032-5

Pfaffenbach, K. T., and Lee, A. S. (2011). The critical Role of GRP78 in physiologic and pathologic stress. Curr. Opin. Cel Biol. 23, 150-156. doi:10.1016/ j.ceb.2010.09.007

Qin, C., Zhou, L., Hu, Z., Zhang, S., Yang, S., Tao, Y., et al. (2020). Dysregulation of Immune Response in Patients with Coronavirus 2019 (COVID-19) in Wuhan, China. Clin. Infect. Dis. 71, 762-768. doi:10.1093/cid/ciaa248

Reckzeh, E. S., and Waldmann, H. (2020). Development of Glucose Transporter (GLUT) Inhibitors. Eur. J. Org. Chem. 2020 (16), 2321-2329. doi:10.1002/ ejoc.2019013510.1002/ejoc.201901353

Sabirli, R., Koseler, A., Goren, T., Turkcuer, I., and Kurt, O. (2021). High GRP78 levels in COVID-19 infection: a case-control study. Life Sci. 265, 118781. doi:10.1016/l.lfs.2020.11878110.1016/j.lfs.2020.118781

Saleh, J., Peyssonnaux, C., Singh, K. K., and Edeas, M. (2020). Mitochondria and microbiota dysfunction in COVID-19 pathogenesis. Mitochondrion 54, 1-7. doi:10.1016/j.mito.2020.06.008

Sasaki, K., Nishina, S., Yamauchi, A., Fukuda, K., Hara, Y., Yamamura, M., et al. (2021). Nanoparticle-mediated delivery of 2-deoxy-D-glucose induces antitumor immunity and cytotoxicity in liver tumors in mice. Cell Mol. Gastroenterol. Hepatol. 11, 739-762. doi:10.1016/j.jcmgh.2020.10.010

Schultheiss, C., Paschold, L., Simnica, D., Mohme, M., Willscher, E., von Wenserski, L., et al. (2020). Next-generation sequencing of T and B cell Receptor Repertoires from COVID-19 patients showed signatures associated with severity of disease. Immunity 53, 442-e4. doi:10.1016/ j.immuni.2020.06.024

Seheult, J., Fitzpatrick, G., and Boran, G. (2017). Lactic acidosis: an Update. Clin. Chem. Lab. Med. 55 (3), 322-333. doi:10.1515/cclm-2016-0438

Serebrovska, Z. O., Chong, E. Y., Serebrovska, T. V., Tumanovska, L. V., and Xi, L. (2020). Hypoxia, HIF-1 $\alpha$, and COVID-19: from pathogenic factors to potential therapeutic targets. Acta Pharmacol. Sin 41 (12), 1539-1546. doi:10.1038/ s41401-020-00554-8

Singh, D., Banerji, A. K., Dwarakanath, B. S., Tripathi, R. P., Gupta, J. P., Mathew, T. L., et al. (2005). Optimizing Cancer Radiotherapy with 2-Deoxy-D-Glucose. Strahlenther Onkol 181, 507-514. doi:10.1007/s00066-005-z10.1007/s00066005-1320-z

Sinha, P., Matthay, M. A., and Calfee, C. S. (2020). Is a "Cytokine Storm" Relevant to COVID-19?. JAMA Intern. Med. 180 (9), 1152-1154. doi:10.1001/ jamainternmed.2020.3313

Strachecka, A., Grzybek, M., Ptaszynska, A. A., Los, A., Chobotow, J., and Rowinski, R. (2019). Comparison of Lactate Dehydrogenase Activity in Hive and Forager Honeybees May Indicate Delayed Onset Muscle Soreness Preliminary Studies. Biochem. Mosc. 84 (4), 435-440. doi:10.1134/ S0006297919040114

Suhail, S., Zajac, J., Fossum, C., Lowater, H., McCracken, C., Severson, N., et al. (2020). Role of Oxidative Stress on SARS-CoV (SARS) and SARS-CoV-2 (COVID-19) Infection: A Review. Protein J. 39 (6), 644-656. doi:10.1007/ s10930-020-09935-8

Taher, A., Lashgari, M., Sedighi, L., Rahimi-Bashar, F., Poorolajal, J., and Mehrpooya, M. (2021). A pilot study on intravenous N-Acetylcysteine treatment in patients with mild-to-moderate COVID19-associated acute Respiratory distress syndrome. Pharmacol. Rep., 1-10. doi:10.1007/s43440021-00296-2

Tang, X., Du, R.-H., Wang, R., Cao, T.-Z., Guan, L.-L., Yang, C.-Q., et al. (2020). Comparison of Hospitalized Patients with ARDS Caused by COVID-19 and H1N1. Chest 158 (1), 195-205. doi:10.1016/j.chest.2020.03.032
Tian, S., Liu, H., Liao, M., Wu, Y., Yang, C., Cai, Y., et al. (2020). Analysis of Mortality in Patients with COVID-19: Clinical and Laboratory Parameters. Open Forum Infect. Dis. 7 (5), 7. doi:10.1093/ofid/ofaa152

Vanderheiden, A., Ralfs, P., Chirkova, T., Upadhyay, A. A., Zimmerman, M. G., Bedoya, S., et al. (2020). Type I and type III interferons Restrict SARS-CoV-2 infection of human airway epithelial cultures. J. Virol. 94, e00985-20. doi:10.1128/JVI.00985-20

Verma, A., Adhikary, A., Woloschak, G., Dwarakanath, B. S., and Papineni, R. V. L. (2020). A combinatorial approach of a polypharmacological adjuvant 2-deoxyD-glucose with low dose Radiation therapy to quell the cytokine storm in COVID-19 management. Int. J. Radiat. Biol. 96 (11), 1323-1328. doi:10.1080/ 09553002.2020.1818865

Wong, K. K., Lee, S. W. H., and Kua, K. P. (2021). N-Acetylcysteine as Adjuvant Therapy for COVID-19 - A Perspective on the Current State of the Evidence. Jir Vol. 14, 2993-3013. doi:10.2147/JIR.S306849

Woodward, G. E., and Cramer, F. B. (1952). 2-Desoxyl-D-glucose as an inhibitor of anaerobic glycolysis in tumor tissue. J. Franklin Inst. 254, 259-260. doi:10.1016/ 0016-0032(52)90482-1

Yang, W.-H., Park, H., Grau, M., and Heine, O. (2020). Decreased Blood Glucose and Lactate: Is a Useful Indicator of Recovery Ability in Athletes?. Ijerph 17 (15), 5470. doi:10.3390/ijerph17155470

Ye, Q., Wang, B., and Mao, J. (2020). The pathogenesis and treatment of the 'Cytokine Storm' in COVID-19. J. Infect. 80, 607-613. doi:10.1016/ j.jinf.2020.03.037

Zhang, H., Penninger, J. M., Li, Y., Zhong, N., and Slutsky, A. S. (2020). Angiotensin-converting enzyme 2 (ACE2) as a SARS-CoV-2 Receptor: molecular mechanisms and potential therapeutic target. Intensive Care Med. 46 (4), 586-590. doi:10.1007/s00134-020-05985-9

Zhang, Q., Ju, Y., Ma, Y., and Wang, T. (2018). N-acetylcysteine improves oxidative stress and inflammatory Response in patients with community acquired pneumonia: a Randomized controlled trial. Medicine (Baltimore) 97, e13087-1541. MD.0000000000013087

Zhang, W., Wang, G., Xu, Z.-G., Tu, H., Hu, F., Dai, J., et al. (2019). Lactate is a Natural suppressor of RLR signaling by targeting MAVS. Cell 178, 176-189. e.115. doi:10.1016/j.cell.2019.05.003

Zhang, Z.-L., Hou, Y.-L., Li, D.-T., and Li, F.-Z. (2020). Laboratory findings of COVID-19: a systematic Review and meta-analysis. Scand. J. Clin. Lab. Invest. 80 (6), 441-447. doi:10.1080/00365513.2020

Zhu, N., Zhang, D., Wang, W., Li, X., Yang, B., Song, J., et al. (2020). A Novel Coronavirus from Patients with Pneumonia in China, 2019. N. Engl. J. Med. 382 (8), 727-733. doi:10.1056/NEJMoa2001017

Conflict of Interest: The authors declare that the research was conducted in the absence of any commercial or financial relationships that could be construed as a potential conflict of interest.

Publisher's Note: All claims expressed in this article are solely those of the authors and do not necessarily represent those of their affiliated organizations, or those of the publisher, the editors and the reviewers. Any product that may be evaluated in this article, or claim that may be made by its manufacturer, is not guaranteed or endorsed by the publisher.

Copyright (C) 2021 Santos, Póvoa, Paixão, Mendonça and Taborda-Barata. This is an open-access article distributed under the terms of the Creative Commons Attribution License (CC BY). The use, distribution or reproduction in other forums is permitted, provided the original author(s) and the copyright owner(s) are credited and that the original publication in this journal is cited, in accordance with accepted academic practice. No use, distribution or reproduction is permitted which does not comply with these terms. 